\title{
A SMOOTHING TECHNIQUE FOR REDUCED INTEGRATION PENALTY METHODS IN CONTACT PROBLEMS
}

\author{
NOBORU KIKUCHI \\ The University of Michigan, Ann Arbor, Michigan, U.S.A.
}

\begin{abstract}
SUMMARY
The paper presents a smoothing technique to the oscillated contact pressure obtained by penalty methods for a class of unilateral contact problems in linear elasticity. The main result is to show that the smoothed contact pressure satisfies the so-called Babuska-Brezzi condition which dominates the convergence of the penalty method. One numerical example is described.
\end{abstract}

\section{INTRODUCTION}

Penalty methods have recently obtained popularity to be applied in the field of finite element methods, to solve constrained problems in mechanics such as Stokes' flow and plate bending problems. ${ }^{1}$ It is also widely admitted that intexact integration must be performed for evaluation of the penalty term in finite element approximation to get physically meaningful solutions. ${ }^{2,3}$ This technique is called reduced integration, and is deeply investigated by Malkus and Hughes ${ }^{4}$ in connection with mixed finite element methods. Within this paper, such a method will be referred to as the reduced integration penalty (RIP) method.

As shown in References 5 and 6, the RIP method is also applicable for solving a class of contact problems. There, convergence of the RIP approximation is proved only for some choice of quadrature rules for the penalty term. If Gaussian quadrature rules, which are most popular, are applied, contact pressure is oscillated, and convergence of the methods cannot be obtained. If trapezoid and Simpson's type quadrature rules, which imply inexact integration of the penalty term, are applied, then contact pressure is smooth, and moreover convergence of the approximation is proved.

In this paper we shall obtain a smoothing technique for oscillated contact pressure which also provides convergence of the method of approximation. Specifically we shall consider the case that the penalty term for unilateral contact constraint will be integrated by one-point Gaussian quadrature rule. This integration rule gives a piecewise constant contact pressure which is oscillated, and does not satisfy the so-called Babuska-Brezzi condition, which describes the constraint qualification condition to the corresponding Lagrangian multiplier in the theory of optimization, and is indispensable to prove convergence of the RIP method. The smoothing technique introduced here gives a piecewise linear contact pressure, which satisfies the Babuska-Brezzi condition, and which is constructed from the solution obtained by using one-point Gaussian quadrature rule. We shall also give a priori error estimates of the RIP method following the analysis in Reference 5, together with a numerical example which confirms the results obtained in the theoretical arguments. 


\section{UNILATERAL CONTACT PROBLEMS}

A typical constrained problem resolving by penalty methods is a unilateral contact problem in elasticity formulated by the variational inequality

$$
u \in K: a(u, v-u) \geqslant f(v-u), \forall v \in K
$$

Here

$$
\begin{aligned}
a(u, v) & =\int_{\Omega} E_{i j k h} u_{k, h} v_{i, j} \mathrm{~d} x \\
f(v) & =\int_{\Omega} f_{i} v_{i} \mathrm{~d} x+\int_{\Gamma_{F}} t_{i} v_{i} \mathrm{~d} s \\
K & =\left\{v \in V: v_{n}-g \leqslant 0 \text { on } \Gamma_{C}\right\}
\end{aligned}
$$

and

$$
V=\left\{v \in\left(H^{1}(\Omega)\right)^{N}: v=0 \text { on } \Gamma_{D}\right\}
$$

The bilinear form $a(.,$.$) represents the virtual work of the elastic body \Omega$ by a virtual displacement $v$ at the equilibrium configuration given by the displacement $u ; f$ and $t$ are applied body forces and surface tractions on a part $\Gamma_{F}$ of the boundary $\Gamma$; and $E_{i j k h}, 1 \leqslant i, j, k, h \leqslant$ $N$, is the elasticity of the body satisfying the conditions

$$
\begin{aligned}
& E_{i j k h}=E_{k h i j}=E_{i j k h} \\
& \exists m>0: E_{i j k h} X_{k h} X_{i j} \geqslant m X_{i j} X_{i j}, \forall X_{i j}=X_{i i}
\end{aligned}
$$

The elastic body is fixed along the boundary $\Gamma_{D}$, and we assume that the total boundary $\Gamma$ of the body consists of three parts: $\Gamma_{F}, \Gamma_{D}$ and $\Gamma_{C}$. The normal displacement $v_{n}$ is defined as $v_{n}=v . n$, where $n$ is the unit vector outward normal to the boundary. The given function $g$ represents the gap between the elastic body and the rigid foundation. The space $H^{1}(\Omega)$ is the Sobolev space, ${ }^{7}$ in which the virtual work of the elastic body is finite. The inner product and norm of $\left(H^{1}(\Omega)\right)^{N}$ are given as

$$
\left\{\begin{aligned}
(u, v)_{1} & =\int_{\Omega}\left\{u_{i} v_{i}+u_{i, j} v_{i, j}\right\} \mathrm{d} x \\
\|u\|_{1} & =\{(u, u)\}^{1 / 2}
\end{aligned}\right.
$$

As shown in Reference 8, the variational inequality (1) has a unique solution $u \in K$, and is obtained as the limit $\varepsilon \rightarrow 0$ of the sequence $\left\{u_{\varepsilon}\right\}$ constructed by the solution $u_{\varepsilon} \in V$ of the penalized problem to (1):

$$
u_{\varepsilon} \in V: a\left(u_{\varepsilon}, v\right)+\frac{1}{\varepsilon}\left(\beta\left(u_{\varepsilon}\right), v_{n}\right)=f(v), \quad v \in V
$$

Here $(.,$.$) is the inner product for L^{2}\left(\Gamma_{C}\right)$, and $\beta$ is the penalty operator defined by

$$
[\beta(v)](\mathbf{x})=\left(v_{n}-g\right)(\mathbf{x})^{+}
$$

with $\phi^{+}=\sup \{\phi, 0\}, \phi \in \mathbb{R}$. 


\section{PENALTY-FINITE ELEMENT APPROXIMATIONS}

We here consider finite element approximations of the penalized problem (8) for the case $N=2$, i.e. $\Omega$ is an open subset of $\mathbb{R}^{2}$. Let $V_{h}$ be a finite element approximation of the space $V$ defined by (5). Let the operator ' $I$ ' be the quadrature rule evaluating the $L^{2}\left(\Gamma_{C}\right)$ inner product:

$$
I(f, g)=\sum_{e=1}^{E} I_{e}(f, g), I_{e}(f, g)=\sum_{i=1}^{G} w_{i}^{e} f\left(\mathbf{x}_{i}^{e}\right) g\left(\mathbf{x}_{i}^{e}\right)
$$

for functions $f$ and $g$ on $\Gamma_{C}$ which are defined on each integration points. Here $E$ is the number of elements overlapped with the boundary $\Gamma_{C}, w_{i}^{e}$ and $x_{i}^{e}$ are weights and co-ordinates of integration points, respectively. For one-point Gaussian quadrature rule, $G$ becomes equal to 1 , and $\mathbf{x}_{1}^{e}$ is the centroid of edge of the eth element. Applying (10), a finite element approximation of $(8)$ is given by

$$
u_{\varepsilon}^{h} \in V_{h}: a\left(u_{\varepsilon}^{h}, v^{h}\right)+\frac{1}{\varepsilon} I\left(\beta\left(u_{\varepsilon}^{h}\right), v_{n}^{h}\right)=f\left(v^{h}\right), \forall v^{h} \in V_{h}
$$

An approximation of the contact pressure $p_{\varepsilon}^{h}$ is then defined by

$$
p_{\varepsilon}^{h}\left(\mathbf{x}_{i}^{e}\right)=-\frac{1}{\varepsilon} \beta\left(u_{\varepsilon}^{h}\right), p_{\varepsilon}^{h} \in Q_{h}
$$

for the space $Q_{h}$ of all polynomials spanned by Lagrangian interpolation functions associated with each integration point. Typical choices of $G$ and $Q_{h}$ are given in Figure 1. For the case that $G=1$ and $V_{h}$ has linear traces on the boundary, $Q_{h}$ is the set of all piecewise constant functions, and the numerical integration ' $I$ ' can provide exact evaluation to the $L^{2}\left(\Gamma_{C}\right)$ inner product between contact pressure $q^{h}$ and normal displacement $v_{n}^{h}$.

As shown in References 5 and 6, success of the method (11) strongly depends upon the Babuska-Brezzi condition, i.e. existence of a positive constant $\beta>0$ such that

$$
\sup _{v^{h} \in V_{h}} \frac{I\left(q^{h}, v_{n}^{h}\right)}{\left\|v^{h}\right\|_{1}} \geqslant \beta h^{1 / 2}\left\|q^{h}\right\|_{0}
$$

for every $q^{h} \in Q_{h}$, where $\|\cdot\|_{0}$ is the norm on $L^{2}\left(\Gamma_{C}\right)$. The existence of $\beta$ for the cases M3 and M4 which provide conforming space $Q_{h}$ has been shown (see Figure 1). However M1 and M2 do not satisfy the condition (13), and the contact pressure identified by (12) is oscillated.

\section{A SMOOTHING TECHNIQUE}

We shall restrict our discussions to the case M1, i.e. one-point Gaussian quadrature rule is applied to evaluate the penalty term. As noted in above, this scheme does not satisfy the condition (13). We shall further simplify the problem so that the surface $\Gamma_{C}$ is identified with a straight line for a two-dimensional domain $\Omega$ of the elastic body.

Let $q_{e}$ and $v_{e}$ be nodal values of $q^{h}$ and $v_{n}^{h}$ on the line $\Gamma_{C}$, respectively. Then, for the case M1, we have

$$
\left(q^{h}, v_{n}^{h}\right)=I\left(q^{h}, v_{n}^{h}\right)=\sum_{e=1}^{E} q_{e}\left(v_{e}+v_{e+1}\right) h_{e} / 2
$$

for a piecewise constant contact pressure $q^{h}$ and a piecewise linear normal displacement $v_{n}^{h}$, where $h_{e}$ is the length of the segment of an element on $\Gamma_{C}$ shown in Figure 2. Within an edge 


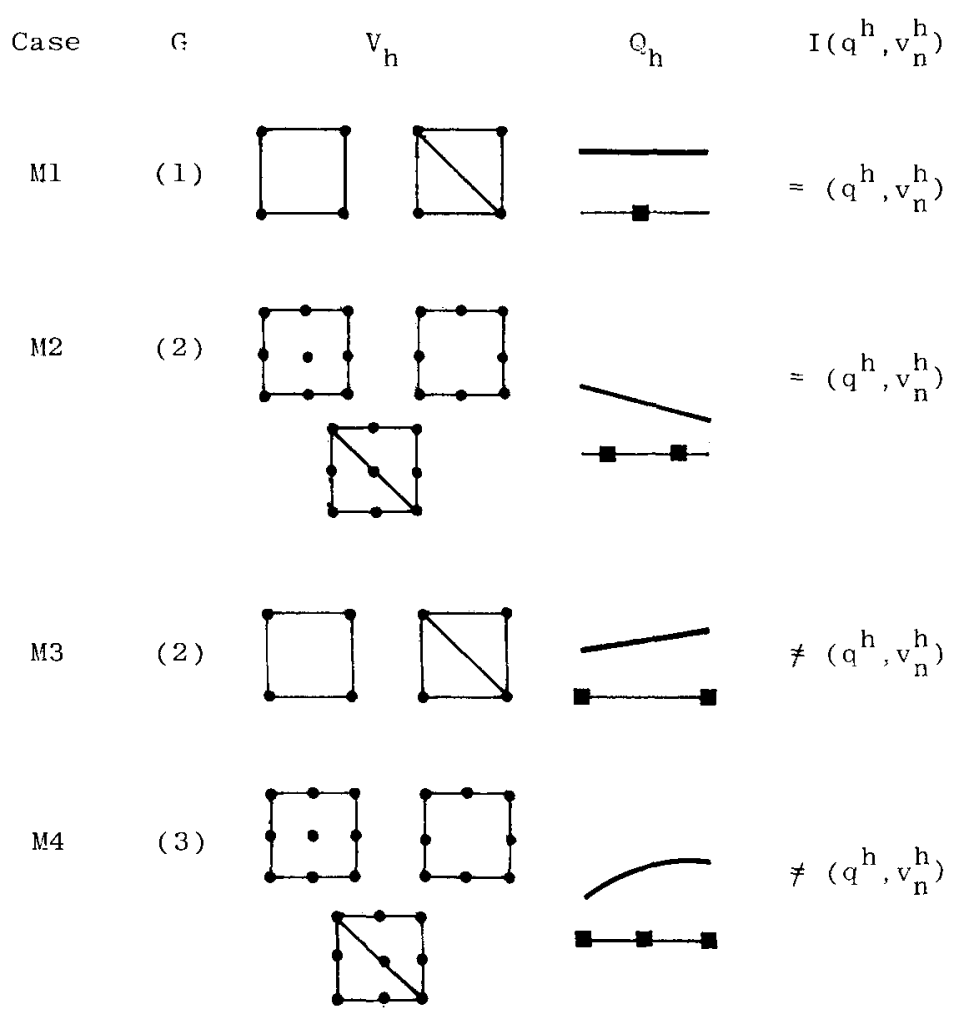

Figure 1. Typical examples of $V_{h}$ and $Q_{h}$
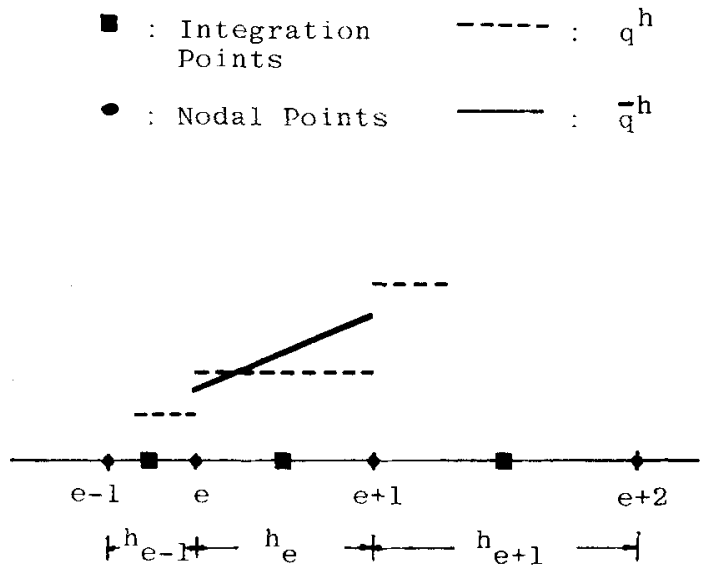

Figure 2. Definitions of $q^{h}$ and $\bar{q}^{h}$ 
of an element we have

$$
\left\{\begin{aligned}
q_{e} & =q^{h}\left(\mathbf{x}_{1}^{e}\right) \\
v_{n}^{h}(\mathbf{x}) & =v_{e}\left(1-\mathbf{x} / h_{e}\right)+v_{e+1} \mathbf{x} / h_{e}
\end{aligned}\right.
$$

Let $\bar{q}^{n}$ be a piecewise linear function defined by

$$
\bar{q}^{h}(\mathbf{x})=\bar{q}_{e}\left(1-\mathbf{x} / h_{e}\right)+\bar{q}_{e+1} \mathbf{x} / h_{e}
$$

where

$$
\left\{\begin{array}{l}
\bar{q}_{1}=q_{1} \quad \text { and } \quad \bar{q}_{E+1}=q_{E} \\
\bar{q}_{e}=\left(h_{e-1} q_{e-1}+h_{e} q_{e}\right) /\left(h_{e-1}+h_{e}\right) e=2, \ldots, E
\end{array}\right.
$$

Then

$$
\begin{aligned}
I\left(q^{h}, v_{n}^{h}\right) & =\bar{q}_{1} v_{1} h_{1} / 2+\sum_{e=2}^{E} \bar{q}_{e} v_{e}\left(h_{e-1}+h_{e}\right) / 2+\bar{q}_{E+1} v_{E+1} h_{E} / 2 \\
& =\sum_{e=1}^{E}\left(\bar{q}_{e} v_{e}+\bar{q}_{e+1} v_{e+1}\right) h_{e} / 2 \\
& =\sum_{e=1}^{E} \hat{I}_{e}\left(\bar{q}^{h}, v_{n}^{h}\right) \\
& =\hat{I}\left(\bar{q}^{h}, v_{n}^{h}\right)
\end{aligned}
$$

where the operator ' $\hat{I}_{e}$ ' is the trapezoid quadrature rule, and

$$
\begin{gathered}
\hat{I}\left(\bar{q}^{h}, v_{n}^{h}\right)=\sum_{e=1}^{E} \hat{I}_{e}\left(\bar{q}^{h}, v_{n}^{h}\right) \\
\hat{I}_{e}\left(\bar{q}^{h}, v_{n}^{h}\right)=\frac{h_{e}}{2}\left(\bar{q}_{e} v_{e}+\bar{q}_{e+1} v_{e+1}\right)
\end{gathered}
$$

This means that the $L^{2}\left(\Gamma_{C}\right)$-inner product between $q^{h}$ and $v_{n}^{h}$ can be identified with the quadrature rule of trapezoid for the $L^{2}\left(\Gamma_{C}\right)$-inner product between piecewise linear functions $\bar{q}^{h}$ and $v_{n}^{h}$. The penalty from (11) is then written as

$$
a\left(u_{\varepsilon}^{h}, v^{h}\right)-\hat{I}\left(\bar{p}_{\varepsilon}^{h}, v_{n}^{h}\right)=f\left(v^{h}\right), \forall v^{h} \in V_{h}
$$

as well as

$$
a\left(u_{\varepsilon}^{h}, v^{h}\right)-\left(p_{\varepsilon}^{h}, v_{n}^{h}\right)=f\left(v^{h}\right), \forall v^{h} \in V_{h} .
$$

Here $\bar{p}_{\varepsilon}^{h}$ is a contact pressure smoothed by (17), and is a piecewise linear polynomial which is continuous on $\Gamma_{C}$.

We next show that $\bar{q}^{h}$ satisfies the LBB condition.

\section{Theorem 1}

Let $\Gamma_{C}$ be a straight line for a two-dimensional domain $\Omega$. Let one-point Gaussian quadrature rule be used to integrate the penalty term, and let ' $\hat{T}$ ' be the trapezoid quadrature rule for 
functions $\bar{q}^{h}$ and $v_{n}^{h}$. Then

$$
\sup _{v^{h} \in V_{h}} \frac{\left(q^{h}, v_{n}^{h}\right)}{\left\|v^{h}\right\|_{1}} \geqslant \beta h^{1 / 2}\left\|\bar{q}^{h}\right\|_{0}
$$

for some positive constant $\beta>0$.

Proof. Taking $v_{e}=\bar{q}_{e}, e=1, \ldots, E+1$, in the form $\left(q^{h}, v_{n}^{h}\right)=\hat{I}\left(\bar{q}^{h}, v_{n}^{h}\right)$ yields

$$
\left(q^{h}, v_{n}^{h}\right)=\left\{\hat{I}\left(\bar{q}^{h}, \bar{q}^{h}\right)\right\}^{1 / 2}\left\{\hat{I}\left(v_{n}^{h}, v_{n}^{h}\right)\right\}^{1 / 2}
$$

By direct expansions, we have

$$
\hat{I}\left(\bar{q}^{h}, \bar{q}^{h}\right) \geqslant\left\|\bar{q}^{h}\right\|_{0}^{2} \quad \text { and } \quad \hat{I}\left(v_{n}^{h}, v_{n}^{h}\right) \geqslant\left\|v_{n}^{h}\right\|_{0}^{2}
$$

Then

$$
\left(q^{h}, v_{n}^{h}\right) \geqslant\left\|\bar{q}^{h}\right\|_{0}\left\|v_{n}^{h}\right\|_{0}
$$

To obtain the inequality $(20)$ it suffices to prove that

$$
\left\|v_{n}^{h}\right\|_{0} \geqslant C\left\|v^{h}\right\|_{1} h^{1 / 2}
$$

for an arbitrary element $v^{h} \in V_{h}$ such that values of $v^{h}$ are zero at all nodal points of the model except on the boundary $\Gamma_{C}$, and the tangential components of $v^{h}$ along $\Gamma_{C}$ vanish. Let $\Gamma_{C}^{e}$ be the edge of the element $\Omega_{e}$ related to the boundary $\Gamma_{C}$. Without loss of generality, we may assume $\Omega_{e}$ is a square whose edges are parallel to the $x-y$ co-ordinate axes. Let $\Gamma_{C}^{e}$ be parallel to the $x$-axis, and let $v_{1}$ and $v_{2}$ be displacements in the $y$-direction (i.e. normal direction) of nodal points located on $\Gamma_{C}^{e}$. If $h$ is the length of edge, we have

$$
\int_{\Gamma_{C}^{e}}\left(v_{n}^{h}\right)^{2} \mathrm{~d} s=\frac{h}{12}\left\{3\left(v_{1}+v_{2}\right)^{2}+\left(-v_{1}+v_{2}\right)^{2}\right\}
$$

and

$$
\int_{\Omega_{e}}\left(v_{i, j}^{h} v_{i, j}^{h}+v_{i}^{h} v_{i}^{h}\right) \mathrm{d} \mathbf{x}=\left(v_{1}+v_{2}\right)^{2}+\frac{5}{3}\left(-v_{1}+v_{2}\right)^{2}+h^{2}\left\{\frac{4}{3}\left(v_{1}+v_{2}\right)^{2}+\frac{4}{9}\left(-v_{1}+v_{2}\right)^{2}\right\} .
$$

Thus, it is clear that there exists a positive constant $C_{e}>0$ such that

$$
\int_{\Gamma \dot{C}}\left(v_{n}^{h}\right)^{2} \mathrm{~d} s \geqslant C_{e} h\left\{\int_{\Omega_{e}}\left(v_{i, j}^{h} v_{i, j}^{h}+v_{i}^{h} v_{i}^{h}\right) \mathrm{d} \mathbf{x}\right\}
$$

Establishing inequalities for each $\Gamma_{C}^{e},(23)$ is now clear.

We have shown that the contact pressure $\bar{q}^{h}$ and $\bar{p}_{\varepsilon}^{h}$, specially constructed by (17) from $q^{h}$ and $p_{\varepsilon}^{h}$, does satisfy the LBB condition which ensures convergence of the method.

Following analysis given in Reference 5, we can also obtain a priori error estimates for the penalty-finite element approximation under a certain regularity assumption on the solution of (1).

Let $V_{h}$ be the finite element approximation of $V$ constructed by $P_{1}$ (three-node triangular) or $Q_{1}$ (four-node quadrilateral isoparametric) elements, and let quadrature in (11) is the one-point Gaussian rule.

\section{Theorem 2}

Let same conditions in Theorem 1 hold, and let

$$
u \in H^{2}(\Omega), p \in H^{1 / 2}\left(\Gamma_{C}\right) \text {, and } g \in H^{3 / 2}\left(\Gamma_{C}\right) \text {. }
$$


Then, the following estimates hold for some positive constants $C_{1}$ and $C_{2}$ independent of the mesh and penalty parameters, $h$ and $\varepsilon$, respectively:

$$
\left\{\begin{array}{l}
\left\|u-u_{\varepsilon}^{h}\right\|_{1} \leqslant C_{1}\left(h+h^{-1 / 4} \varepsilon^{1 / 2}\right) \\
\left\|p-\bar{p}_{\varepsilon}^{h}\right\|_{0} \leqslant C_{2}\left(h^{1 / 2}+h^{-3 / 4} \varepsilon^{1 / 2}\right)
\end{array}\right.
$$

We simply note that a key step to obtain estimates $(26)$ is the condition (21) proved in Theorem 1, and that details of derivation (26) can be found in Reference 5, especially Theorems 6.1, 6.2, 7.1 and Example 6.1 in Reference 5.

\section{NUMERICAL EXAMPLE}

As a model problem we shall consider a plane strain problem of Hertz type. Let $\Omega$ be a isotropic and homogeneous linearly elastic foundation characterized by Young's modulus 1000 and Poisson ratio $0 \cdot 3$, and let $\Omega$ be a rectangular of $4 \times 16$ as shown in Figure 3 . An infinitely long circular cylinder is indented into the centre of the foundation. Let the cylinder be rigid, and let its radius be 8 . We discretize the foundation by $Q_{1}$-elements, and integrate the penalty term by the one-point Gaussian quadrature rule.

Pressure distributions given in Figure 4 are obtained by 32 and $128 Q_{1}$-elements. It is easily observed that the contact pressure $\bar{p}_{\varepsilon}^{h}$ is smooth, whereas $p_{\varepsilon}^{h}$, which is originally obtained by the penalty method, is oscillated.

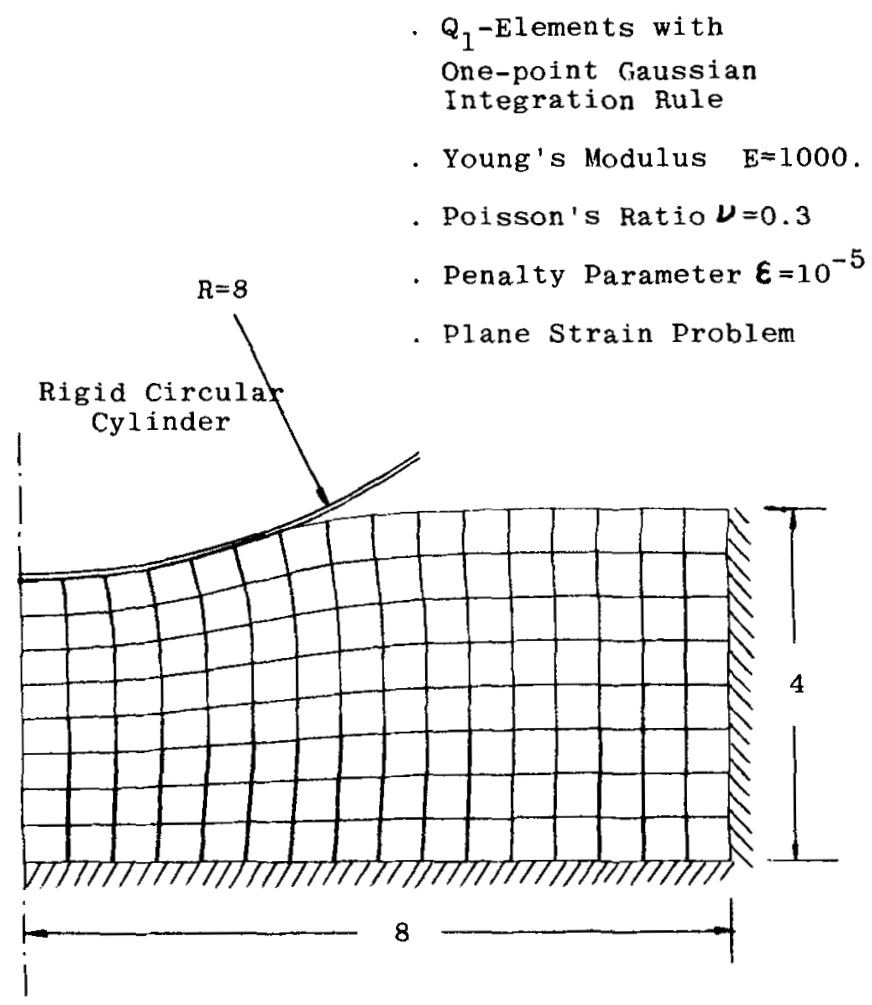

Figure 3. Finite element model and deformed configuration (depth of indentation $=0 \cdot 8$ ) 


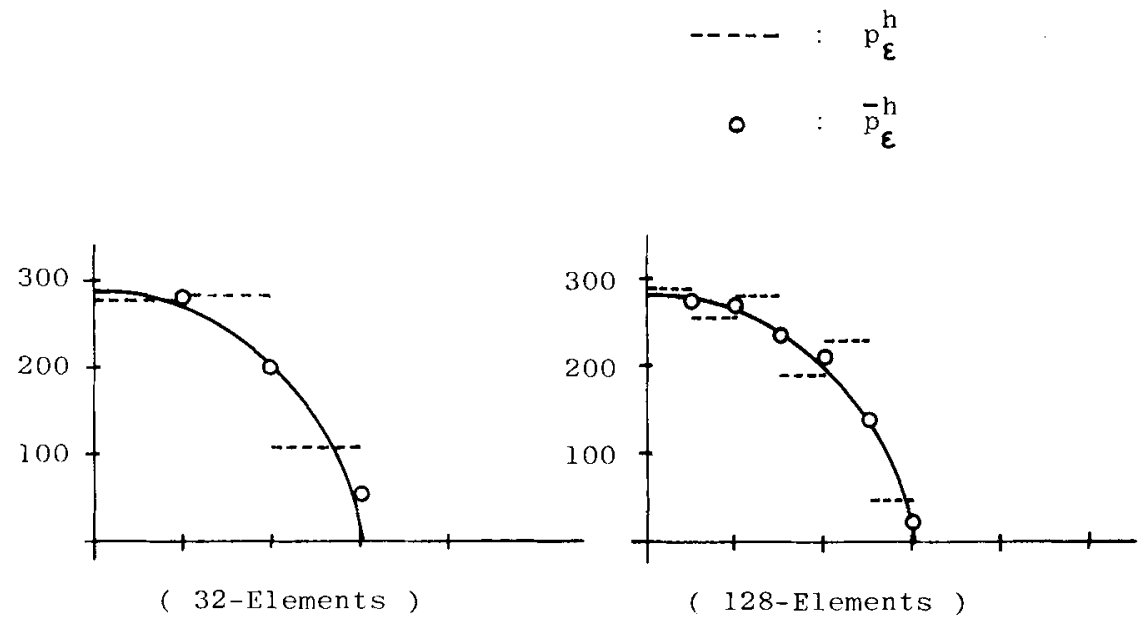

Figure 4. Distributions of contact pressure

\section{REFERENCES}

1. O. C. Zienkiewicz, The Finite Element Method, 3rd edn, McGraw-Hill, ch. 11.

2. O. C. Zienkiewicz, R. L. Taylor and J. M. Too, 'Reduced integration techniques in general analysis of plates and shells', Int. J. num. Meth. Engng, 3, 275-90 (1971).

3. D. J. Naylor, 'Stresses in nearly incompressible materials for finite elements with application to the calculation of excess pore pressures', Int. J. num. Meth. Engng, 8, 443-60 (1974).

4. D. S. Malkus and T. J. R. Hughes, 'Mixed finite element methods-reduced and selective integration technique-a unification of concepts', Comp. Meth. Appl. Mech. Eng. 15 (1978).

5. N. Kikuchi and Y. J. Song, 'Penalty-finite element approximations of a class of unilateral problems in linear elasticity', Q. Appl. Math. (to appear).

6. J. T. Oden, N. Kikuchi and Y. J. Song, 'Reduced integration and exterior penalty methods for finite element approximations of contact problems in incompressible elasticity', TICOM Report $80-2$, The University of Texas at Austin (1980)

7. J. Nečas, Les Methodes Directes en Theorie des Equations Elliptiques, Masson, Paris-VI ${ }^{\mathrm{e}}, 1967$.

8. N. Kikuchi and $\mathcal{H}$. T. Oden, 'Contact problems in elasticity', TICOM Report 79-8, The University of Texas at Austin (1979).

9. P. G. Ciarlet, The Finite Element Method for Elliptic Problems, North-Holland, Amsterdam, 1978. 